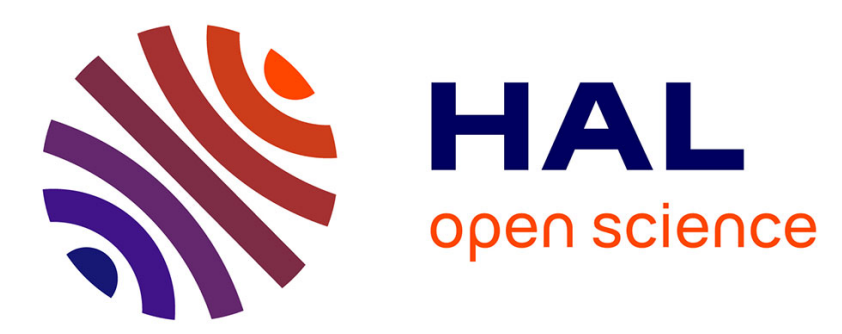

\title{
Spatial Receptor Allocation for a Multiple Access Hub in Nanonetworks
}

Fazilet Gokbudak, Bayram Cevdet Akdeniz, Tuna Tugcu, Ali Emre Pusane

\section{To cite this version:}

Fazilet Gokbudak, Bayram Cevdet Akdeniz, Tuna Tugcu, Ali Emre Pusane. Spatial Receptor Allocation for a Multiple Access Hub in Nanonetworks. IEEE Transactions on Molecular, Biological and Multi-Scale Communications, 2019, 10.1109/TMBMC.2019.2946819 . hal-02415965

\section{HAL Id: hal-02415965 \\ https://hal.inria.fr/hal-02415965}

Submitted on 17 Dec 2019

HAL is a multi-disciplinary open access archive for the deposit and dissemination of scientific research documents, whether they are published or not. The documents may come from teaching and research institutions in France or abroad, or from public or private research centers.
L'archive ouverte pluridisciplinaire HAL, est destinée au dépôt et à la diffusion de documents scientifiques de niveau recherche, publiés ou non, émanant des établissements d'enseignement et de recherche français ou étrangers, des laboratoires publics ou privés. 


\title{
Spatial Receptor Allocation for a Multiple Access Hub in Nanonetworks
}

\author{
Fazilet Gokbudak, Bayram Cevdet Akdeniz, Tuna Tugcu, and Ali Emre Pusane
}

\begin{abstract}
Following the same trends in other communication schemes, design of star network topologies will gain importance in order to communicate with a centralized hub in molecular communications as well. For a molecular communication multiple access network system, interlink interference (ILI) becomes a crucial issue in addition to intersymbol interference (ISI). This paper focuses on spatial allocation of the receptors of a centralized receiver among transmitters by proposing efficient solutions to reduce both ISI and ILI. The spatial allocation scheme has been designed using an optimization problem and verified with Monte Carlo simulations.
\end{abstract}

Index Terms-Molecular Communication via Diffusion, spatial transmitter allocation, nanonetworks

\section{INTRODUCTION}

In the last decade, molecular communication has increased its popularity as a possible solution of the communication of nanomachines [1], [2]. In recent molecular communication literature, different kinds of networks have been examined. These networks can be categorized into two network topologies as star networks and point-to-point networks. In pointto-point networks, main objective is to transmit an intended symbol from source to destination using relay nodes, while in star networks, multiple transmitters try to communicate with a centralized hub.

As point-to-point networks, one-way multi-hop diffusion nanonetworks are considered in [3], and the decode and forward method is proposed to increase the performance of the system. In [4] and [5], network coding is used for twoway communication to increase the performance of the system by increasing the data rate. In [6], an optimal barrier function is derived to minimize the error in point-to-point networks and a routing protocol for the network has been presented in [7].

In the molecular communication literature, star network topologies have also been considered frequently. One of the possible applications of the star topology in nanonetworks can be healthcare monitoring by transmitting the sensed information to a centralized receiver, which can process these transmitted information as proposed in [8]. In [9], the statistical interference model of the channel is derived when multiple nanomachines release molecules to a centralized receiver. In [8], the bit error rate (BER) is optimized by scheduling the transmitters' release time of molecules and adjusting the concentration of these released molecules. In [10], a stochastic model of the network that involves a spherical receiver and randomly placed transmitters whose emission times are also random is derived. In [11], queueing network theory is used to model molecular nanonetworks.
Although there are various works related to nanonetworks with star network topology, to the best of our knowledge, none of them has examined the optimum spatial allocation of the receptors of the centralized receiver among transmitters to optimize communication performance. On the other hand, in relay network topology, optimum spatial position of the relay is determined to improve the performance [12]. In this paper, we propose a method to find the optimal spatial allocation of the receiver's receptors among the transmitters. As proposed in [13], the transmitted molecules are more likely to be absorbed by the receptors that are closer to the transmitter. This is not surprising, since for these closer regions, molecules travel over shorter distances compared to the other regions. Hence, the molecules absorbed by the receptors of the receiver that are far from the transmitter require more time to reach these regions, and they are most likely to belong to the previous symbols, contributing to intersymbol interference (ISI). In [13], it has been proved that the part of the receiver that is far from the transmitter should not be used for decoding. Inspired from this idea, we claim that there is plenty of room at the receiver for other transmitters. In this paper, we propose a method to allocate the parts of the receiver to different transmitters. The main motivation behind this allocation is determining the area of the receptors assigned to each transmitter and also determining the barriers between two consecutive receptor regions assigned to two neighboring transmitters in order to reduce both ISI and interlink interference (ILI).

\section{SySTEM MODEL}

We consider $N$ point nanotransmitters that are evenly placed around a centralized receiver. For the sake of simplicity, we consider $N$ identical channels, but the proposed methods can be modified for different scenarios. Each transmitter aims to send its own message to the centralized spherical absorbing receiver with radius $r_{\mathrm{r}}$ by releasing molecules in the diffusive fluidic channel. As shown in Fig.1, different parts of the receiver surface are allocated for receiving messages from different transmitters. In Fig. 1a, the area of the allocated part of the receiver for each transmitter (also named as patch) is defined by the base of a spherical sector, whose apical angle is $2 \alpha_{\mathrm{r}}$. Furthermore, as seen from the same figure, each pair of consecutive patches are separated by a neutral zone whose apical angle is $\alpha_{\mathrm{s}}$, and the molecules absorbed at these neutral zones are not taken into account by the receiver for decision. In particular, these zones can be considered as barriers between consecutive patches in order to reduce ILI.

It is assumed that each transmitter sends its binary messages by on-off keying, which is a special case of concentration shift 


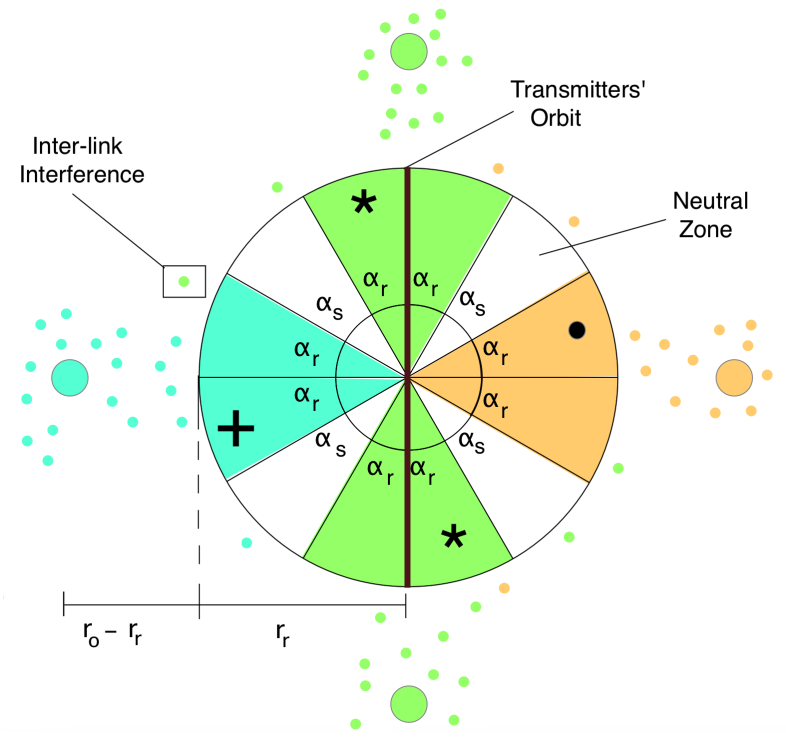

(a) Channel model for $N=6 \mathrm{Tx}$-Rx pair

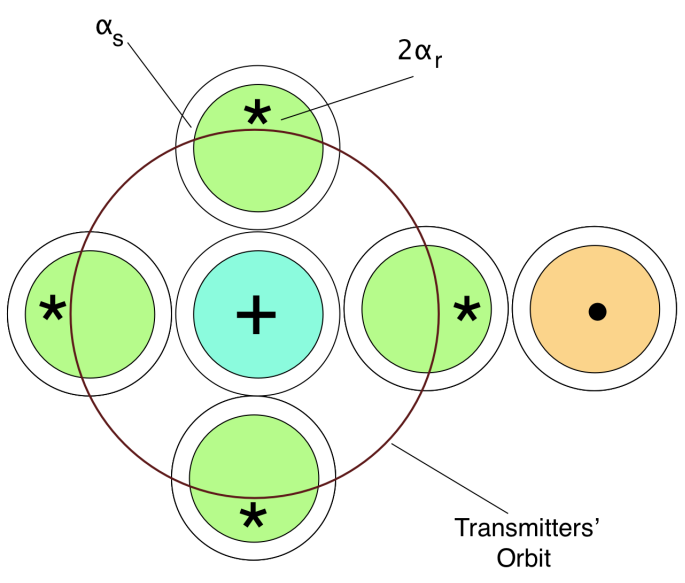

(b) Expanded view of the channel model

Fig. 1: Left: Spatial allocation of the spherical receiver patches by point transmitters. The apical angle for each receiver is $2 \alpha_{\mathrm{r}}$ and there are neutral zones with $\alpha_{\mathrm{s}}$ that separate neighboring receptor parts. Right: Expanded top view of the communication system.

keying. In particular, each transmitter releases $M$ molecules at the beginning of the transmission period $t_{\mathrm{s}}$ for transmitting bit-1 and releases nothing to convey bit- 0 . The molecules in the channel move under the effects of free diffusion, which is explained in [14]. At the end of the transmission period, the receiver makes a decision on the symbols transmitted from the transmitters by using the number of absorbed molecules at each patch.

In order to propose our approaches, a method for evaluating the number of the transmitters (hence, corresponding receivers) for a given $\alpha_{\mathrm{r}}$ and $\alpha_{\mathrm{s}}$ is required. As an illustrative example in Fig.1, assuming that $2 \alpha_{\mathrm{r}}+\alpha_{\mathrm{s}}=\pi / 2$, six transmitters exist for communications. Furthermore, we derive the analytical expression of the number of transmitter-receiver pairs for given $\alpha_{\mathrm{r}}$ and $\alpha_{\mathrm{s}}$. Note that each receiver surface corresponds to a cone, whose apical angle is $2 \alpha_{\mathrm{r}}$. Between all neighbour receivers, there is a $\alpha_{\mathrm{s}}$ gap to eliminate interference. Considering these, we can say that we require an angle of $2 \alpha_{\mathrm{r}}+\alpha_{\mathrm{s}} / 2+\alpha_{\mathrm{s}} / 2=2 \alpha_{\mathrm{r}}+\alpha_{\mathrm{s}}$ for each receiver. Therefore, in order to calculate the area required to place a receiver, we need to calculate the area of the spherical cap that coincides with the base of the cone whose apical angle is $2 \alpha_{\mathrm{r}}+\alpha_{\mathrm{s}}$, which is equal to $2 \pi \mathrm{r}_{\mathrm{r}}^{2}\left(1-\cos \left(\alpha_{\mathrm{r}}+\alpha_{\mathrm{s}} / 2\right)\right)$. It is clear that the upper limit of the number of transmitters for given $\alpha_{\mathrm{r}}$ and $\alpha_{\mathrm{s}}$ can be obtained by dividing the area of the sphere by the area of the spherical cap that coincides with the base of the cone whose apical angle is $2 \alpha_{\mathrm{r}}+\alpha_{\mathrm{s}}$ as

$$
N=\left\lfloor\frac{4 \pi \mathrm{r}_{\mathrm{r}}^{2}}{2 \pi \mathrm{r}_{\mathrm{r}}^{2}\left(1-\cos \left(\alpha_{\mathrm{r}}+\frac{\alpha_{\mathrm{s}}}{2}\right)\right)}\right\rfloor=\left\lfloor\frac{2}{\left(1-\cos \left(\alpha_{\mathrm{r}}+\frac{\alpha_{\mathrm{s}}}{2}\right)\right)}\right\rfloor .
$$

Since each channel is identical (due to symmetry), it is enough to examine the communication performance of only one of them to determine the overall performance. The performance is evaluated using BER, which is affected by ISI due to the remaining molecules released by the transmitter for previous symbols and ILI caused by the molecules released by the neighboring transmitters. Considering the leftmost channel in Fig. 1a, ILI can most possibly occur due to the transmission from neighboring transmitters. Due to the nature of diffusion, amount of ILI coming from the rightmost channel to the leftmost channel is very limited compared to other channels. Therefore, the number of neighbors for any patch, $N_{1}$, for a given $\alpha_{\mathrm{r}}$ and $\alpha_{\mathrm{s}}$ pair must be determined. In order to calculate $N_{1}$, we need to define the transmitter orbit, which is a hypothetical circle, and all patches assigned to these neighboring transmitters are lined up on this orbit as shown in Fig. 1a and 1b. Considering Fig. 1a, one can easily compute the radius of this orbit $r_{\text {or }}$ as

$$
r_{\text {or }}=r_{\mathrm{r}} \sin \left(2 \alpha_{\mathrm{r}}+\alpha_{\mathrm{s}}\right) \text {. }
$$

Therefore, the patches are lined up to a circle whose circumference is $2 \pi r_{\text {or }}$. Accordingly, the number of patches placed in this orbit $\left(N_{1}\right)$ can be obtained by dividing this circumference to the length of the arc of any patch that coincides with the orbit. Since the angle of the arc is $\left(2 \alpha_{\mathrm{r}}+\alpha_{\mathrm{s}}\right)$, as can be seen in Fig. 1b, the arclength can be calculated as $r_{\mathrm{r}}\left(2 \alpha_{\mathrm{r}}+\alpha_{\mathrm{s}}\right)$. Therefore, $N_{1}$ can be written as

$$
N_{1}=\left\lfloor\frac{2 \pi r_{\mathrm{r}} \sin \left(2 \alpha_{\mathrm{r}}+\alpha_{\mathrm{s}}\right)}{r_{\mathrm{r}}\left(2 \alpha_{\mathrm{r}}+\alpha_{\mathrm{s}}\right)}\right\rfloor=\left\lfloor\frac{2 \pi \sin \left(2 \alpha_{\mathrm{r}}+\alpha_{\mathrm{s}}\right)}{\left(2 \alpha_{\mathrm{r}}+\alpha_{\mathrm{s}}\right)}\right\rfloor .
$$

It is possible that there can be many orbits satisfying $2 k \alpha_{\mathrm{r}}+$ $k \alpha_{\mathrm{s}} \leq \pi / 2$ (for example, for the second closest neighbour receivers, the radius of the orbit would be $\left.r_{\mathrm{r}} \sin \left(4 \alpha_{\mathrm{r}}+2 \alpha_{\mathrm{s}}\right)\right)$. 
Therefore, one can calculate the number of transmitters at the $k-t h$ orbit as

$$
N_{k}=\left\lfloor\frac{2 \pi r_{\mathrm{r}} \sin \left(2 k \alpha_{\mathrm{r}}+k \alpha_{\mathrm{s}}\right)}{r_{\mathrm{r}}\left(2 \alpha_{\mathrm{r}}+\alpha_{\mathrm{s}}\right)}\right\rfloor=\left\lfloor\frac{2 \pi \sin \left(2 \alpha_{\mathrm{r}}+\alpha_{\mathrm{s}}\right)}{\left(2 \alpha_{\mathrm{r}}+\alpha_{\mathrm{s}}\right)}\right\rfloor .
$$

Hence, considering Fig. 1a, $2 \alpha_{\mathrm{r}}+\alpha_{\mathrm{s}}=\pi / 2$, there is only one transmitter orbit, and the number of patches at this orbit can be obtained using (4) as $\lfloor 2 \pi \sin (\pi / 2) /(\pi / 2)\rfloor=4$, which can also be verified by Fig.1b. Once the number of neighboring transmitters (hence patches) is derived, the next step is to determine the fraction of absorbed molecules by the patch to the molecules released from the assigned transmitter to this patch at the $n-t h$ slot $\left(p_{n}\right)$ and the fraction of absorbed molecules released from the neighbors of this transmitter to the molecules released from the assigned transmitter $\left(l_{n}\right)$. In other words, $p_{n}$ and $l_{n}$ correspond to ISI and ILI, respectively, while $p_{1}$ is related to the intended signal. In [13], the cumulative distribution of absorbed molecules by a spherical receiver until time $t$ and angle $\alpha(F(\alpha, t))$ has already been derived. Using this function, the channel taps can be computed as

$$
\begin{aligned}
& p_{n}\left(\alpha_{\mathrm{r}}, t_{\mathrm{s}}\right)=F\left(\alpha_{\mathrm{r}}, n t_{\mathrm{s}}\right)-F\left(\alpha_{\mathrm{r}},(n-1) t_{\mathrm{s}}\right) \\
& l_{n}\left(\alpha_{\mathrm{r}}, \alpha_{\mathrm{s}}, t_{\mathrm{s}}\right)=\left(F\left(3 \alpha_{\mathrm{r}}+\alpha_{\mathrm{s}}, n t_{\mathrm{s}}\right)-F\left(3 \alpha_{\mathrm{r}}+\alpha_{\mathrm{s}},(n-1) t_{\mathrm{s}}\right)\right) \\
& -\left(F\left(\alpha_{\mathrm{r}}+\alpha_{\mathrm{s}}, n t_{\mathrm{s}}\right)-F\left(\alpha_{\mathrm{r}}+\alpha_{\mathrm{s}},(n-1) t_{\mathrm{s}}\right)\right) / N_{1} .
\end{aligned}
$$

Note that $l_{n}\left(\alpha_{\mathrm{r}}, \alpha_{\mathrm{s}}, t_{\mathrm{s}}\right)$ corresponds to the ILI taps that correspond to the channel closest to the corresponding channel, whose channel taps are defined by $p_{n}\left(\alpha_{\mathrm{r}}, t_{\mathrm{s}}\right)$.

\section{Optimum Spatial Allocation}

In this paper, we investigate the optimum spatial allocation of the receiver to transmitters for two cases. The first one is the case where the number of transmitters, $N$, is fixed. For the second case, the total data rate transmitted to the receiver is fixed. In particular, the number of allowed bit transmissions per second is fixed and the number of transmitters is also an additional variable for this second problem. For both cases, our aim is to determine $\alpha_{\mathrm{r}}$ and $\alpha_{\mathrm{s}}$ angles that achieve the lowest BER. BER function in molecular communication channel can be obtained by considering all possible transmitted sequences, hence involves $2^{L}$ terms, where $L$ is the channel memory. In multiple access channels, one also needs to take ILI into account by considering the sequences that the neighboring transmitters send. All in all, BER involves $2^{L N_{1}}$ terms, and it is not a tractable function. [15] proposes an objective function named signal-to-interference difference (SID), which is basically $p_{1}-\sum_{n=2}^{L} p_{n}$ and provides approximately the same angles that BER gives. Inspired from this function, we propose the modified version of this function for multiple access channels. Since ILI is another factor that affects the performance of the system, signal-to-inter-symbol and -link interference difference function (SILD) is proposed to determine optimized $\alpha_{\mathrm{r}}$ and $\alpha_{\mathrm{s}}$ values as

$$
\begin{aligned}
\operatorname{SILD}\left(\alpha_{\mathrm{r}}, \alpha_{\mathrm{s}}, t_{\mathrm{s}}\right)= & p_{1}\left(\alpha_{\mathrm{r}}, t_{\mathrm{s}}\right)-\sum_{n=2}^{L} p_{n}\left(\alpha_{\mathrm{r}}, t_{\mathrm{s}}\right) \\
& -\sum_{n=2}^{L} l_{n}\left(\alpha_{\mathrm{r}}, \alpha_{\mathrm{s}}, t_{\mathrm{s}}\right)
\end{aligned}
$$

\section{A. Optimum Spatial Allocation for a Fixed Number of Trans- mitters}

In the first case, it is assumed that there is a fixed number of transmitters $(N)$ that transmit their symbols during a fixed symbol interval $t_{\mathrm{s}}$, and the aim is to determine $\alpha_{\mathrm{r}}$ and $\alpha_{\mathrm{s}}$ to obtain the lowest BER or highest SILD. Since $N$ is fixed, it can be concluded that $2 \alpha_{\mathrm{r}}+\alpha_{\mathrm{s}}$ is also constrained by (1). Considering this constraint, the optimization problem is reduced to finding either $\alpha_{\mathrm{r}}$ or $\alpha_{\mathrm{s}}$ by achieving maximum SILD as

$$
\begin{aligned}
& \alpha_{\mathrm{r}}^{*}=\underset{\alpha_{\mathrm{r}}}{\operatorname{argmax}} \operatorname{SILD}\left(\alpha_{\mathrm{r}}, \alpha_{\mathrm{s}}, t_{\mathrm{s}}\right) \\
& \text { subject to: } \quad N<\frac{2}{\left(1-\cos \left(\alpha_{\mathrm{r}}+\alpha_{\mathrm{s}} / 2\right)\right)}<N+1 .
\end{aligned}
$$

\section{B. Optimum Spatial Allocation for Fixed Data Rate}

When the number of bits transmitted per second, namely data rate $(R)$, is fixed, the number of transmitters becomes an additional variable for the optimization problem. In this case, for any $\alpha_{\mathrm{r}}$ and $\alpha_{\mathrm{s}}$, the number of transmitters $N$ can be obtained as given in (1). Therefore, in order to achieve $R$ bits per second, the required transmission symbol duration $\left(t_{\mathrm{s}}^{\text {req }}\right)$ can be obtained as

$$
t_{\mathrm{s}}^{\mathrm{req}}=R / N
$$

Since the data rate $(R)$ is fixed, the total number of transmitters $N$ and the required symbol duration to achieve this data rate $t_{\mathrm{s}}^{\text {req }}$ are constraint variables. Therefore, considering (7), the constraint turns out to be $R=t_{\mathrm{s}}^{\text {req }}\left\lfloor 2 /\left(1-\cos \left(\alpha_{\mathrm{r}}+\alpha_{\mathrm{s}} / 2\right)\right)\right\rfloor$. This constraint implies that, unlike the optimization problem defined in (7), there are two parameters that optimize the SILD, which are $v=\left\{\alpha_{\mathrm{s}}, \alpha_{\mathrm{r}}\right\}$. Therefore, the optimization problem for fixed data rate can be written as

$$
\begin{aligned}
& v^{*}=\underset{\alpha_{\mathrm{r}}, \alpha_{\mathrm{s}}}{\operatorname{argmax}} \operatorname{SILD}\left(\alpha_{\mathrm{r}}, \alpha_{\mathrm{s}}, t_{\mathrm{s}}^{\mathrm{req}}\right) \\
& \text { subject to: } \quad R=t_{\mathrm{s}}^{\text {req }}\left\lfloor\frac{2}{\left(1-\cos \left(\alpha_{\mathrm{r}}+\alpha_{\mathrm{s}} / 2\right)\right)}\right\rfloor .
\end{aligned}
$$

\section{Performance Analysis}

Although the analytical expression of SILD can be obtained by using the results in [13], the corresponding SILD function is so complicated that it is not tractable to obtain an analytical solution. Therefore, performance analysis of the proposed systems is done by solving the optimization problems defined 


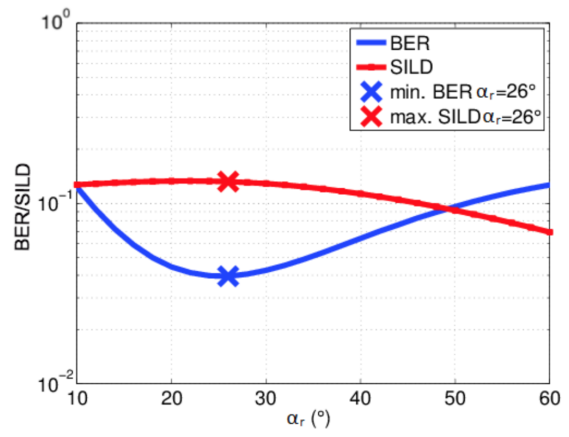

(a) for $t_{\mathrm{s}}=100 \mathrm{~ms}$ and $N=2$ users

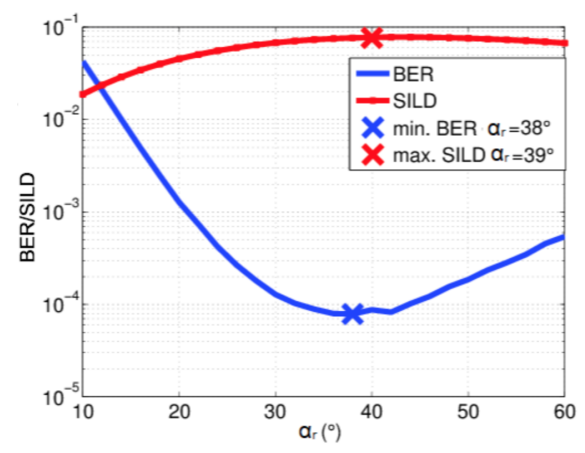

(d) for $t_{\mathrm{s}}=200 \mathrm{~ms}$ and $N=2$ users

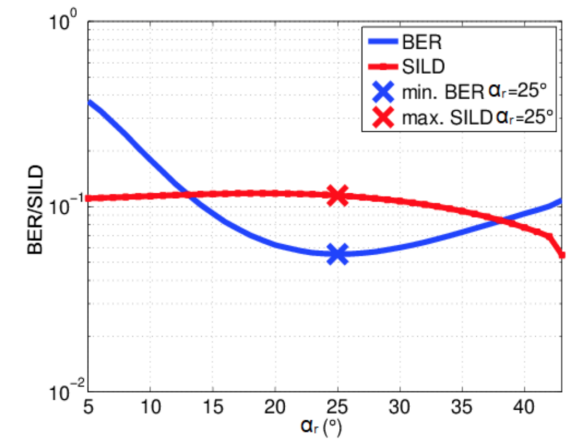

(b) for $t_{\mathrm{s}}=100 \mathrm{~ms}$ and $N=6$ users

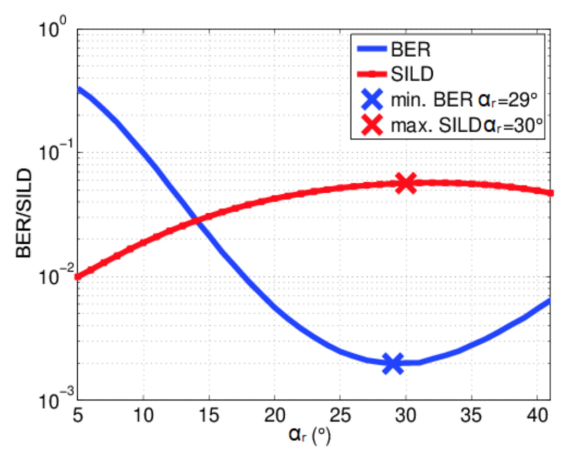

(e) for $t_{\mathrm{s}}=200 \mathrm{~ms}$ and $N=6$ users

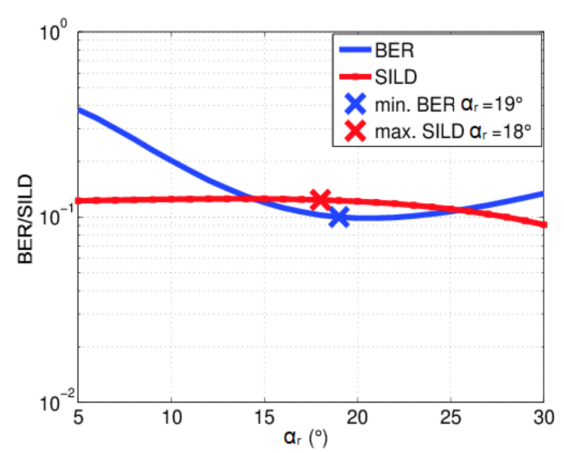

(c) for $t_{\mathrm{s}}=100 \mathrm{~ms}$ and $N=14$ users

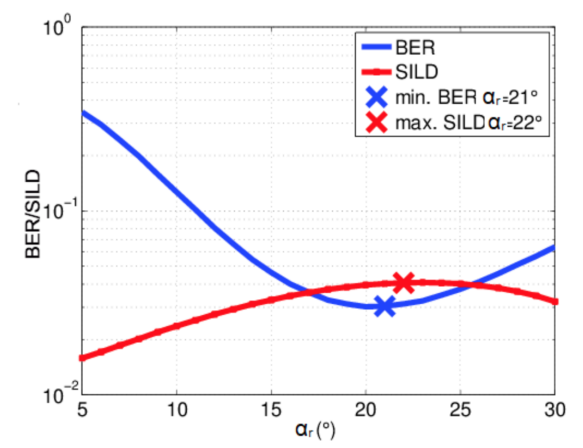

(f) for $t_{\mathrm{s}}=200 \mathrm{~ms}$ and $N=14$ users

Fig. 2: Optimum $\alpha_{\mathrm{r}}$ to obtain lowest BER and maximum SILD

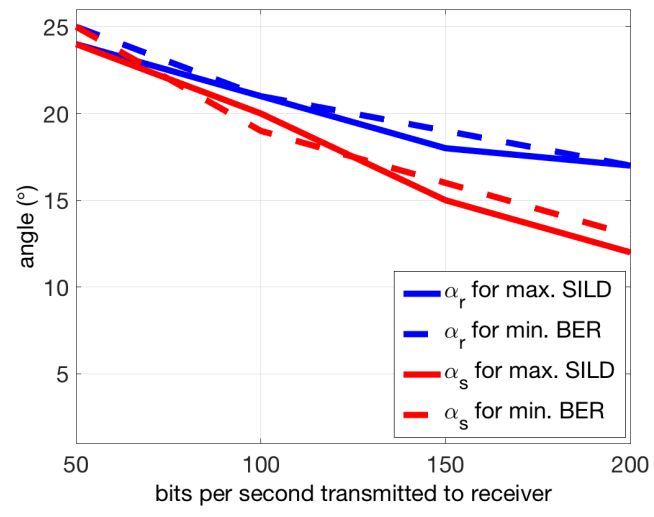

Fig. 3: Optimum $\alpha_{\mathrm{r}}$ and $\alpha_{\mathrm{s}}$ vs. data rate

in (7) and (9) numerically and is also verified by obtaining the lowest BER with exhaustive search to show that the angle parameters of the minimum BER and maximum SILD are approximately the same.

In order to evaluate BER, once the channel taps are obtained from [13], normal approximations are done to model the number of arriving molecules to the receiver as done in [16]. In particular, to transmit bit-1, the transmitter releases $M$ molecules and the arrival of these molecules can be modelled by using the channel taps, $p_{n}$. Similarly, for modelling the arrival of the molecules from the neighbour channels, same methodology can be used by utilizing $l_{n}$. For BER simulations, the threshold is always chosen to obtain the lowest BER in order to determine the ultimate performance of the systems. The performed simulations are conducted for $10^{4}$ times and, for each simulation, $10^{5}$ consecutive binary symbols are transmitted in a channel with memory $L=100$, and $M$ is chosen as 500 molecules.

It is observed from Fig. 2 that as the number of users $N$ increases, the optimum angle $\alpha_{\mathrm{r}}$ decreases. This is expected since the allocated region to each transmitter should be narrower for more users. Furthermore, it can also be seen that the angle parameters of the minimum BER and maximum SILD are almost the same, which enables us to find the optimum receptor allocation scheme using SILD optimization without exhaustive BER simulations.

In Fig. 3, the optimization problem defined in (9) is examined for various data rates. In particular, as can be seen in (9), the data rate is considered as the amount of data transferred to the receiver. Therefore, both the number of transmitters $N$ and corresponding transmission slot $t_{\mathrm{s}}^{\mathrm{req}}$ are not constant, and they are easily determined by finding the optimum $\alpha_{\mathrm{r}}$ and $\alpha_{\mathrm{s}}$. One can observe in Fig. 3 that, as the data rate increases, both $\alpha_{\mathrm{r}}$ and $\alpha_{\mathrm{s}}$ decrease. The decrease in $\alpha_{\mathrm{r}}$ and $\alpha_{\mathrm{s}}$ are expected since, as the amount of data transferred to the receiver increases, more transmitters are required and when the number of transmitters is increased, we need to compress them in smaller patches, which implies smaller $\alpha_{\mathrm{r}}$ and $\alpha_{\mathrm{s}}$ values. Furthermore, $\alpha_{\mathrm{r}}$ and $\alpha_{\mathrm{s}}$ values obtained by the proposed SILD function yield almost the same values with minimum BER obtained using exhaustive search. 


\section{CONCLUSION}

In this paper, a star network communication scheme for nanonetworks is proposed for reducing the ISI and ILI. The proposed scheme is based on the allocation of the absorbing surface of the receiver (patch) to transmitters by assigning a neutral zone between neighboring receivers. In particular, the proposed approach consists of determining the surface area of the patch assigned to each transmitter as well as neutral zone. The optimization problems are constructed to determine the optimum surface area for each patch, and the results are verified with simulations that show that the solution of the proposed optimization problem yields minimum BER without performing exhaustive simulations for BER calculations.

\section{REFERENCES}

[1] I. F. Akyildiz, F. Brunetti, and C. Blazquez, "Nanonetworks: A new communication paradigm," Elsevier Comput. Netw., vol. 52, no. 12, pp. 2260-2279, Aug. 2008.

[2] N. Farsad, H. B. Yilmaz, A. Eckford, C.-B. Chae, and W. Guo, "A comprehensive survey of recent advancements in molecular communication," IEEE Communications Surveys \& Tutorials, vol. 18, no. 3, pp. 1887-1919, 2016

[3] A. Ahmadzadeh, A. Noel, and R. Schober, "Analysis and design of multi-hop diffusion-based molecular communication networks," IEEE Transactions on Molecular, Biological and Multi-Scale Communications, vol. 1, no. 2, pp. 144-157, 2015.

[4] B. C. Akdeniz, B. Tepekule, A. E. Pusane, and T. Tugcu, "Novel network coding approaches for diffusion-based molecular nanonetworks," Transactions on Emerging Telecommunications Technologies, vol. 28, no. 7, 2017.

[5] A. Aijaz, A. Aghvami, and M. Nakhai, "On error performance of network coding in diffusion-based molecular nanonetworks," IEEE Transactions on Nanotechnology, vol. 13, no. 5, pp. 871-874, 2014.

[6] S. K. Tiwari, T. R. T. Reddy, and P. K. Upadhyay, "Error performance optimization using logarithmic barrier function in molecular nanonetworks," IEEE Communications Letters, vol. 21, no. 11, pp. 2408-2411, 2017.

[7] A. Aijaz, "Opportunistic routing in diffusion-based molecular nanonetworks," IEEE wireless communications letters, vol. 4, no. 3, pp. 321$324,2015$.

[8] M. J. Moore, Y. Okaie, and T. Nakano, "Diffusion-based multiple access by nano-transmitters to a micro-receiver," IEEE Communications Letters, vol. 18, no. 3, pp. 385-388, 2014.

[9] M. Pierobon and I. F. Akyildiz, "A statistical-physical model of interference in diffusion-based molecular nanonetworks," IEEE Transactions on communications, vol. 62, no. 6, pp. 2085-2095, 2014.

[10] F. Zabini, "Spatially distributed molecular communications: An asynchronous stochastic model," IEEE Communications Letters, 2018.

[11] V. Honary, T. A. Wysocki, and B. J. Wysocki, "Modeling molecular channel using queueing theory approach," in 2017 IEEE International Conference on Electro Information Technology, EIT 2017. IEEE Computer Society, 2017.

[12] N. Tavakkoli, P. Azmi, and N. Mokari, "Optimal positioning of relay node in cooperative molecular communication networks," IEEE Transactions on Communications, vol. 65, no. 12, pp. 5293-5304, 2017.

[13] B. C. Akdeniz, N. A. Turgut, H. B. Yilmaz, C.-B. Chae, T. Tugcu, and A. E. Pusane, "Molecular signal modeling of a partially counting absorbing spherical receiver," IEEE Transactions on Communications, vol. 66, no. 12, pp. 6237-6246, 2018.

[14] H. B. Yilmaz, A. C. Heren, T. Tugcu, and C.-B. Chae, "Threedimensional channel characteristics for molecular communications with an absorbing receiver," Communications Letters, IEEE, vol. 18, no. 6, pp. 929-932, 2014

[15] B. C. Akdeniz, A. E. Pusane, and T. Tugcu, "Optimal reception delay in diffusion-based molecular communication," IEEE Communications Letters, vol. 22, no. 1, pp. 57-60, 2018.

[16] H. B. Yilmaz and C.-B. Chae, "Arrival modelling for molecular communication via diffusion," Electronics Letters, vol. 50, no. 23, pp. 16671669, 2014. 